\title{
NOTES ON F-THEORY COMPACTIFICATIONS
}

\author{
TERUHIKO KAWANO \\ Department of Physics, University of Tokyo, \\ Tokyo 113-0033, Japan \\ kawano@hep-th.phys.s.u-tokyo.ac.jp
}

\begin{abstract}
We study the Kähler potential of charged matter fields, whose profiles have a peak on their matter curve - on an "intersection" of 7-branes, in an F-theory compactification. It is shown that the Kähler potential is exactly given by the integral over the matter curve, but not by the integral over the whole GUT surface of 7-branes. This talk is based on our paper "A Note on Kahler Potential of Charged Matter in F-theory," in collaboration with Yoichi Tsuchiya and Taizan Watari.
\end{abstract}

Keywords: String theory; F-theory; string compactifications.

PACS Numbers: 11.25.Hf, 11.25.Wx

\section{Introduction}

To understand the flavor structure in the standard model of elementary particle physics is a big challenge. Especially when one may consider a supersymmetric phenomenological model in order to resolve the hierarchy problem, the Kähler potential in the visible sector has important consequences in physics. For example, the higher terms suppressed by the Planck mass scale in the Kähler potential contains important couplings between particles in the visible sector and and messenger fields in a supersymmetry breaking scenario to understand the FCNC problem and the $\mathrm{CP}$ violation. However, it seems formidable to determine those couplings from the point of view of the low-energy effective theory, and string theory is virtually the only theoretical framework at least at present which enables us to make some theoretical progress. $^{1}$

To this end, one may prefer to consider a string compactification which is as phenomenological viable as possible. In particular, it would be interesting to study the flavor problems of the gravity mediated supersymmetry breaking scenario in such a compacitification where the flavor violation in the Yukawa couplings has already been understood.

An F-theory compactification with charged matter fields of the supersymmetric Standard Model arising from matter curves is one of such compactifications. After a few years of intense study on the flavor structure of the Yukawa couplings, in fact, it is now known that the realistic flavor pattern can be realized in the compactification, 
either by tuning one or a few complex structure parameters of the matter curve of the matter fields, ${ }^{2}$ or by taking an appropriate factorization limit ${ }^{3,4}$ of geometry. ${ }^{\text {a }}$ See Refs. 2 and 11 for yet another solution using discrete symmetries.

In an F-theory compactification, a charged matter field appears on a "7-brane intersection" called a matter curve, ${ }^{12}$ and is given by a holomorphic section of a line bundle on the matter curve. ${ }^{13-17}$ In terms of the worldvolume theory of 7-branes carrying gauge fields of the GUT gauge group, which is called the GUT divisor, the wavefunction of the charged matter field is given by the zero-mode solution to the equation of motion of the worldvolume theory, and in fact localizes on the matter curve with a decaying profile away from the curve.

Although the wavefunction is defined on the GUT divisor, which is a surface, Refs. 4 and 18 have exploited the BPS nature of the superpotential and the residue integral technique to show that the Yukawa couplings of charged matter fields in the superpotential are given by their holomorphic sections on the matter curves.

In this talk, ${ }^{20}$ we will show that the residue integral of Refs. 19, 4 and 18 can also be used to show that the Kähler potential of charged matter fields on matter curves is localized on the matter curves.

In this talk, we will illustrate our idea about the localization in a simple setting. See our original paper ${ }^{20}$ for more realistic setting and more complete list of references.

\section{Kähler Potential of Charged Matter on Matter Curve}

The bosonic fields in the low-energy effective theory on the worldvolume of 7-branes in an F-theory compactification are an eight-dimensional gauge field consisting of four-dimensional external part $A_{\mu}(\mu=0,1,2,3)$, and the internal part

$$
A=A_{m} d z^{m}, \quad \bar{A}=\bar{A}_{\bar{m}} d z^{\bar{m}}
$$

along the GUT divisor $S$ with the local complex coordinates $z^{m=1,2}$, and a two-form field

$$
\Phi=\frac{1}{2} \Phi_{m n} d z^{m} \wedge d z^{n}
$$

in the adjoint representation of the gauge group $G$. Henceforth, we will take the gauge group $G$ to be $S U(N)$ for simplicity.

\footnotetext{
${ }^{\text {a This is to avoid }}{ }^{2,5,6}$ multiple points of the enhanced singularities of the $E_{6}$ type and the $D_{6}$ type in the $\mathrm{SU}(5)_{\mathrm{GUT}} \mathrm{GUT}$ divisor contributing to the low-energy up-type and down-type Yukawa matrices. It should be noted that the factorization of a spectral surface is not enough as discussed in Refs. $7-10$.
} 
In terms of the field strengths of the internal gauge field $A$

$$
\begin{aligned}
& F_{A \mu}^{(0,1)}=\partial_{\mu} \bar{A}-\bar{\partial} A_{\mu}+\left[A_{\mu}, \bar{A}\right], \quad F_{A}^{(0,2)}=\bar{\partial} \bar{A}+\bar{A} \wedge \bar{A}, \\
& F_{A}^{(1,1)}=\partial \bar{A}+\bar{\partial} A+A \wedge \bar{A}+\bar{A} \wedge A,
\end{aligned}
$$

the bosonic part of the action is given by

$$
\begin{aligned}
& -\int d^{4} x \int_{S} \operatorname{tr}\left[\frac{1}{2} \omega \wedge \omega\left(-\frac{1}{4} F_{\mu \nu}^{2}+\frac{1}{2} D^{2}\right)+D_{\mu} \Phi^{\dagger} D^{\mu} \Phi-i \omega \wedge F_{A \mu}^{(1,0)} \wedge F_{A}^{(0,1) \mu}\right. \\
& -D\left(\left[\Phi, \Phi^{\dagger}\right]+i \omega \wedge F_{A}^{(1,1)}\right)-2 F_{A}^{(2,0)} \wedge F_{A}^{(0,2)}+2 i \omega \wedge \bar{G} \wedge G \\
& \left.\quad+2 \bar{G} \wedge \partial_{A} \Phi^{\dagger}+2 \bar{\partial}_{A} \Phi \wedge G\right],
\end{aligned}
$$

with the Kähler form $\omega$ of the GUT divisor $S$, where $D$ and $G=G_{\bar{m}} d z^{\bar{m}}$ are auxiliary fields. The last four terms in the action (1) can be wrapped up into the superpotential

$$
W=\int_{S} \operatorname{tr}\left[F_{A}^{(0,2)} \wedge \Phi\right]
$$

From the superpotential, one can read the F-term condition

$$
F_{A}^{(0,2)}=\bar{\partial} \bar{A}+\bar{A} \wedge \bar{A}=0, \quad \bar{\partial}_{A} \Phi=\bar{\partial} \Phi+[\bar{A}, \Phi]=0 .
$$

It is known that a solution to them is given in term of a matrix $V$ taking values in $G L(N, \mathbf{C})$ and an $N \times N$ matrix $\phi_{m n}$ by

$$
\bar{A}=V \bar{\partial} V^{-1}, \quad \Phi=V \phi V^{-1},
$$

where the two-form $\phi$ satisfies

$$
\bar{\partial} \phi=0 .
$$

Under an $S U(N)$ gauge transformation with a unitary matrix $U$, they transform as

$$
V \mapsto U \cdot V, \quad \phi \mapsto \phi .
$$

A redundancy of this parameterization

$$
V \mapsto V \cdot \Lambda
$$

where $\Lambda$ is a matrix taking values in $G L(N, \mathbf{C})$ and is holomorphic; $\bar{\partial} \Lambda=0$, can be regarded as the origin of a holomorphic vector bundle.

Using the hermitian metric $H=V^{\dagger} V$ of the holomorphic vector bundle, the D-term condition

$$
\omega \wedge F_{A}^{(1,1)}=i\left[\Phi, \Phi^{\dagger}\right]
$$

may be rewritten as

$$
\omega \wedge \bar{\partial}\left(H^{-1} \mathrm{H}\right)=i\left[\phi, H^{-1} \phi^{\dagger} H\right]
$$

where $A=-(\bar{A})^{\dagger}$. Therefore, a solution $(V, \phi)$ to the D-term condition serves as a supersymmetric background in the worldvolume theory. 
About the supersymmetric background $(V, \phi)$, let us consider a fluctuation $(\bar{\alpha}, \varphi)$ given by

$$
\bar{A}=V \bar{\partial} V^{-1}+\bar{a}=V \bar{\partial} V^{-1}+V \bar{\alpha} V^{-1}, \quad \Phi=V \phi V^{-1}+V \varphi V^{-1} .
$$

The F-term condition (2) for them yields

$$
\bar{\partial} \bar{\alpha}=0, \quad \bar{\partial} \varphi+[\bar{\alpha}, \phi]=0 .
$$

The first equation of (5) can be solved locally by

$$
\bar{\alpha}=\bar{\partial} v
$$

with a function $v$, and there is a redundancy in the definition of $v$ by a holomorphic function $\epsilon$ as

$$
v \rightarrow v+\epsilon \text {. }
$$

The second equation of (5) is then solved in terms of a holomorphic function $h$ as

$$
\varphi=h+[\phi, v],
$$

and due to the redundancy (6), the holomorphic function $h$ also transforms as

$$
h \rightarrow h-[\phi, \epsilon],
$$

which, with (6), let us call a holomorphic shift transformation.

For a constant $N \times N$ matrix $N$, suppose that there exists a constant $N \times N$ matrix $M$ such that they satisfy

$$
[\phi, N]=f M,
$$

with a non-constant holomorphic function $f$. Since a component $\zeta$ of $h=\zeta M$ transforms under the holomorphic shift transformation as

$$
\zeta \mapsto \zeta+f \varepsilon,
$$

with $\epsilon=\varepsilon N$, one may regard the component $\zeta$ as a mode essentially localized on the curve $\Sigma: f=0$, as discussed in Ref. 18. By introducing $\eta=\zeta N$, one may also rewrite $h$ as

$$
h=\left[\phi, \frac{\eta}{f}\right] .
$$

This is the description of localized modes in the holomorphic gauge given by Ref. 18 .

In order to describe the localized mode (7) completely, one needs to obtain a solution of $v$ to the equation

$$
\begin{aligned}
\omega \wedge & \left(H \cdot \bar{\partial} v \cdot H^{-1}\right)+i\left[\phi^{\dagger}, H \varphi H^{-1}\right] \\
& =H \cdot\left\{-\omega \wedge \bar{\partial}\left(H^{-1} \cdot \partial v^{\dagger} \cdot H\right)+i\left[\phi, H^{-1} \varphi^{\dagger} H\right]\right\} \cdot H^{-1},
\end{aligned}
$$

which comes from the D-term condition $(3)$, and the resulting pair $(v, \eta)$ gives rise to a massless mode in the four-dimensional low-energy effective theory. 
We would like to study the kinetic term of the localized modes $(7)$ in the fourdimensional low-energy effective theory. To this end, one needs to substitute the localized modes

$$
\bar{A}=V \bar{\partial} V^{-1}+\sum_{i} \chi_{i}\left(x^{\mu}\right) \cdot V \cdot \bar{\partial} v_{i} \cdot V^{-1}, \quad \Phi=V\left(\phi+\sum_{i} \chi_{i}\left(x^{\mu}\right) \cdot \varphi_{i}\right) V^{-1},
$$

located on curves $\Sigma_{i}: f_{i}=0$, respectively, with a four-dimensional field $\chi_{i}\left(x^{\mu}\right)$, into the kinetic term

$$
\int d^{4} x^{\mu} \int \operatorname{tr}\left[-D_{\mu} \Phi^{\dagger} D^{\mu} \Phi+i \omega \wedge F_{A}^{(1,0)}{ }_{\mu} \wedge F_{A}^{(0,1) \mu}\right]
$$

in the 7 -brane worldvolume action (1), and one finds the kinetic term

$$
-\sum_{i, j} \int d^{4} x^{\mu} \mathcal{N}_{i j} \partial_{\mu} \chi_{i} \partial^{\mu} \chi_{j}
$$

where the normalization $\mathcal{N}_{i j}$ is given by

$$
\mathcal{N}_{i j}=\int \operatorname{tr}\left[\left(h_{i}^{\dagger}+\left[v_{i}^{\dagger}, \phi^{\dagger}\right]\right) H \wedge\left(h_{j}+\left[\phi, v_{j}\right]\right) H^{-1}+i \omega \wedge \partial v_{i}^{\dagger} \cdot H \wedge \bar{\partial} v_{j} \cdot H^{-1}\right] .
$$

Assuming that the localized modes $\left(v_{i}, h_{i}\right)$ satisfies the condition

$$
\omega \wedge \partial\left(H \cdot \bar{\partial} v_{i} \cdot H^{-1}\right)+i\left[\phi^{\dagger}, H\left(h_{i}+\left[\phi, v_{i}\right]\right) H^{-1}\right]=0
$$

which is the left hand side of (8), one may then note that the normalization $\mathcal{N}_{i j}$ may be rewritten as

$$
\begin{aligned}
\mathcal{N}_{i j}= & \int \operatorname{tr}\left[h_{i}^{\dagger} \wedge H\left(h_{j}+\left[\phi, v_{j}\right]\right) H^{-1}+\left(i \omega \wedge v_{i}^{\dagger} H \bar{\partial} v_{j} \cdot H^{-1}\right)\right. \\
& \left.-v_{i}^{\dagger}\left\{i \omega \wedge \partial\left(H \bar{\partial} v_{j} \cdot H^{-1}\right)-\left[\phi^{\dagger}, H\left(h_{j}+\left[\phi, v_{j}\right]\right) H^{-1}\right]\right\}\right] \\
= & \int \operatorname{tr}\left[h_{i}^{\dagger} \wedge H\left(h_{j}+\left[\phi, v_{j}\right]\right) H^{-1}+\left(i \omega \wedge v_{i}^{\dagger} H \bar{\partial} v_{j} \cdot H^{-1}\right)\right] .
\end{aligned}
$$

Since for the localized modes (7), one finds that

$$
\begin{aligned}
\operatorname{tr}\left[h_{i}^{\dagger}\right. & \left.\wedge H\left(h_{j}+\left[\phi, v_{j}\right]\right) H^{-1}\right]=\operatorname{tr}\left[\frac{\eta_{i}^{\dagger}}{\bar{f}_{i}}\left[\phi^{\dagger}, H\left(h_{j}+\left[\phi, v_{j}\right]\right) H^{-1}\right]\right] \\
& =i \omega \wedge \operatorname{tr}\left[\frac{\eta_{i}^{\dagger}}{\bar{f}_{i}} \cdot \partial\left(H \bar{\partial} v_{j} \cdot H^{-1}\right)\right] \\
& =\operatorname{tr}\left[\eta_{i}^{\dagger} \partial\left(\frac{1}{\bar{f}_{i}}\right) \wedge\left(-i \omega \wedge H \bar{\partial} v_{j} \cdot H^{-1}\right)\right]+\partial \operatorname{tr}\left[i \omega \wedge \frac{\eta_{i}^{\dagger}}{\bar{f}_{i}} \cdot H \bar{\partial} v_{j} \cdot H^{-1}\right] .
\end{aligned}
$$

Using the fact that

$$
\partial\left(\frac{1}{\bar{f}}\right)=-i \pi \delta^{2}(f) d f
$$


one can see that the normalization $\mathcal{N}_{i j}$ may be given by

$$
\begin{aligned}
\mathcal{N}_{i j}= & \int \operatorname{tr}\left[-\pi \delta^{2}\left(f_{i}\right) d f_{i} \wedge\left(\omega \wedge \eta_{i}^{\dagger} \cdot H \bar{\partial} v_{j} \cdot H^{-1}\right)\right. \\
& \left.+\partial\left(i \omega \wedge\left(v_{i}^{\dagger}+\frac{\eta_{i}^{\dagger}}{\bar{f}_{i}}\right) H \bar{\partial} v_{j} \cdot H^{-1}\right)\right] .
\end{aligned}
$$

Since the combination $v_{i}^{\dagger}+\left(\eta_{i}^{\dagger} / \bar{f}_{i}\right)$ is left invariant under a holomorphic shift transformation, it should be globally defined, and the second term of the above equation may be dropped as a surface term over $S$, which is assumed to be compact. One thus finds that the normalization $\mathcal{N}_{i j}$ is given by the integral over the matter curve $\Sigma_{i}: f_{i}=0$;

$$
\mathcal{N}_{i j}=-\pi \int \delta^{2}\left(f_{i}\right) d f_{i} \wedge \omega \wedge \operatorname{tr}\left[\eta_{i}^{\dagger} \cdot H \bar{\partial} v_{j} \cdot H^{-1}\right] .
$$

Incidentally, for the (massless) localized modes $\left(v_{i}, \eta_{i}\right)$, the superpotential

$$
W=\int \operatorname{tr}\left[F_{A}^{(0,2)} \wedge \Phi\right]
$$

gives Yukawa couplings

$$
\sum_{i, j, k} Y_{i j k} \chi_{i}\left(x^{\mu}\right) \chi_{j}\left(x^{\mu}\right) \chi_{k}\left(x^{\mu}\right)
$$

where the coupling constants $Y_{i j k}$ is given ${ }^{4,18}$ by

$$
\begin{aligned}
Y_{i j k}= & \int \operatorname{tr}\left[\bar{a}_{i} \wedge \bar{a}_{j} \wedge V \varphi_{k} V^{-1}\right]=\int \operatorname{tr}\left[\bar{\partial} v_{i} \wedge \bar{\partial} v_{j} \wedge \varphi_{k}\right] \\
= & -\int \bar{\partial}\left(\frac{1}{f_{i}}\right) \wedge \operatorname{tr}\left[\eta_{i} \bar{\partial} v_{j} \wedge \varphi_{k}\right]+\int \bar{\partial} \operatorname{tr}\left[\left(v_{i}+\frac{\eta_{i}}{f_{i}}\right) \wedge \bar{\partial} v_{j} \wedge \varphi_{k}\right] \\
= & \int \bar{\partial}\left(\frac{1}{f_{i}}\right) \wedge \bar{\partial}\left(\frac{1}{f_{j}}\right) \wedge \operatorname{tr}\left[\eta_{i} \eta_{j} \varphi_{k}\right] \\
& +\int \bar{\wedge}\left(\bar{\partial}\left(\frac{1}{f_{i}}\right) \wedge \operatorname{tr}\left[\eta_{i}\left(v_{j}+\frac{\eta_{j}}{f_{j}}\right) \wedge \varphi_{k}\right]+\operatorname{tr}\left[\left(v_{i}+\frac{\eta_{i}}{f_{i}}\right) \wedge \bar{\partial} v_{j} \wedge \varphi_{k}\right]\right) \\
= & -\pi^{2} \int \delta^{2}\left(f_{i}\right) \delta^{2}\left(f_{j}\right) \operatorname{tr}\left[\eta_{i} \eta_{j} \varphi_{k}\right] \wedge d \bar{f}_{i} \wedge d \bar{f}_{j} \\
= & -\pi^{2} \int \delta^{2}\left(f_{i}\right) \delta^{2}\left(f_{j}\right) \operatorname{tr}\left[\eta_{i} \eta_{j} h_{k}\right] \wedge d \bar{f}_{i} \wedge d \bar{f}_{j},
\end{aligned}
$$

in the last equality of which we assumed that $f_{k}=0$ at a point where $f_{i}=f_{j}=0$. 
The superpotential also gives the couplings of the localized modes $\left(v_{i}, \eta_{i}\right)$ with the bulk modes $\bar{\alpha}_{b}, \varphi_{b}$ as

$$
\int \operatorname{tr}\left[\bar{\alpha}_{i} \wedge \bar{\alpha}_{b} \wedge \varphi_{j}\right]+\int \operatorname{tr}\left[\bar{\alpha}_{b} \wedge \bar{\alpha}_{i} \wedge \varphi_{j}\right]
$$

and

$$
\int \operatorname{tr}\left[\bar{\alpha}_{i} \wedge \bar{\alpha}_{j} \wedge \varphi_{b}\right]
$$

the former of which may be rewritten as

$$
\begin{gathered}
\int \operatorname{tr}\left[\bar{\alpha}_{i} \wedge \bar{\alpha}_{b} \wedge \varphi_{j}\right]+\int \operatorname{tr}\left[\bar{\alpha}_{b} \wedge \bar{\alpha}_{i} \wedge \varphi_{j}\right]=-\int \bar{\partial}\left(\frac{1}{f_{i}}\right) \wedge \operatorname{tr}\left[\left[\eta_{i}, \bar{\alpha}_{b}\right] \wedge \varphi_{j}\right] \\
=\int \bar{\partial}\left(\frac{1}{f_{i}}\right) \wedge \operatorname{tr}\left[h_{j} \wedge\left[\bar{\alpha}_{b}, \eta_{i}\right]\right]=-i \pi \int \delta^{2}\left(f_{i}\right) d \bar{f}_{i} \wedge \operatorname{tr}\left[h_{j} \wedge\left[\bar{\alpha}_{b}, \eta_{i}\right]\right]
\end{gathered}
$$

where we have replaced $\varphi_{j}$ by $h_{j}$, because we have assumed that it gives no contributions if $f_{i} \neq f_{j}$. The latter coupling

$$
\int \operatorname{tr}\left[\bar{\alpha}_{i} \wedge \bar{\alpha}_{j} \wedge \varphi_{b}\right]=-\int \bar{\partial}\left(\frac{1}{f_{i}}\right) \wedge \operatorname{tr}\left[\eta_{i} \wedge \bar{\alpha}_{j} \wedge \varphi_{b}\right]
$$

gives no contributions, if $\bar{\alpha}_{j}$ is propotional to $d \bar{f}_{i}$ at $\Sigma_{i}=0$. Since the bulk mode $\varphi_{b}$ is a $(2,0)$-form on the 7-brane worldvolume, the pull-back of it onto the matter curve $\Sigma$ should be zero, and there are expected to be no couplings of the bulk mode $\varphi_{b}$ with the localied matters in the low-energy effective action.

We will apply the above considerations to the intersecting brane background in the $S U(2)$ gauge theory on the worldvolume of 7 -branes. The intersecting brane background defined by

$$
\phi=\frac{1}{2}\left(\begin{array}{cc}
x & 0 \\
0 & -x
\end{array}\right), \quad H=\mathbf{1}
$$

with the local coordinates $\left(z^{1}, z^{2}\right)=(x, y)$, obviously satisfies the D-term condition (4). Here, note that we have omitted $d x \wedge d y$ in $\phi$ for the notational simplicity. One can easily see that localized modes $h_{ \pm}(y)$ on the curve $\Sigma: x=0$ are given by

$$
h_{+}(y)=\left(\begin{array}{cc}
0 & \zeta_{+}(y) \\
0 & 0
\end{array}\right), \quad h_{-}(y)=\left(\begin{array}{cc}
0 & 0 \\
\zeta_{-}(y) & 0
\end{array}\right),
$$

and one can also notice that $h_{ \pm}(y)= \pm \eta_{ \pm}(y)$. We will concentrate on the localized mode $h_{+}(y)$, because the same discussions are applied to the other mode $h_{-}(y)$. In this case, the equation (8) is equivalent to (9), and a solution to it with a flat metric

$$
\omega=i h_{x \bar{x}} d x \wedge d \bar{x}+i h_{y \bar{y}} d y \wedge d \bar{y}
$$


is known ${ }^{12}$ to be

$$
v_{+}=h_{+}(y) \frac{1}{x}\left(e^{-\sqrt{\frac{1}{h_{y \bar{y}}}}|x|^{2}}-1\right)
$$

Since one has

$$
\left.\bar{\alpha}_{+}\right|_{x=0}=\left.\bar{\partial} v_{+}\right|_{x=0}=-\sqrt{\frac{1}{h_{y \bar{y}}}} h_{+}(y) d \bar{x}
$$

one obtains the normalization $\mathcal{N}_{+}$as

$$
\mathcal{N}_{+}=\int \pi \operatorname{tr}\left[h_{+}^{\dagger} h_{+}\right] \sqrt{h_{y \bar{y}}} i d y \wedge d \bar{y}=\pi \int\left|\zeta_{+}(y)\right|^{2} \sqrt{h_{y \bar{y}}} i d y \wedge d \bar{y}
$$

The bulk modes $\bar{\alpha}_{b}$ of $\bar{A}$ are give by

$$
\bar{\alpha}_{b}(x, y)=\frac{1}{\sqrt{2}}\left(\begin{array}{cc}
\bar{\alpha}_{3} & 0 \\
0 & -\bar{\alpha}_{3}
\end{array}\right)
$$

and the couplings of the localized modes $\left(v_{ \pm}, \eta_{ \pm}\right)$with the bulk modes yields

$$
\begin{aligned}
-i \pi & \int \delta^{2}(x) d \bar{x} \wedge\left[\operatorname{tr}\left[h_{+} \wedge\left[\bar{\alpha}_{b}, \eta_{-}\right]\right]+\operatorname{tr}\left[h_{-} \wedge\left[\bar{\alpha}_{b}, \eta_{+}\right]\right]\right] \\
& =-i \pi \int \delta^{2}(x) d \bar{x} \wedge\left[\operatorname{tr}\left[-h_{+} \wedge\left[\bar{\alpha}_{b}, h_{-}\right]\right]+\operatorname{tr}\left[h_{-} \wedge\left[\bar{\alpha}_{b}, h_{+}\right]\right]\right] \\
& =-2 \sqrt{2} i \pi \int \delta^{2}(x) d \bar{x} \wedge \zeta_{+} \bar{\alpha}_{3} \zeta_{-} d x \wedge d y=2 \sqrt{2} \pi \int \zeta_{+} \bar{\alpha}_{3 \bar{y}} \zeta_{-} i d y \wedge d \bar{y}
\end{aligned}
$$

and

$$
\begin{gathered}
\int \operatorname{tr}\left[\left(\bar{\alpha}_{+} \wedge \bar{\alpha}_{-}+\bar{\alpha}_{-} \wedge \bar{\alpha}_{+}\right) \wedge \varphi_{b}\right]=\int \operatorname{tr}\left[\left(\bar{\alpha}_{+} \wedge \bar{\partial} v_{-}+\bar{\partial} v_{-} \wedge \bar{\alpha}_{+}\right) \wedge \varphi_{b}\right] \\
=-\int \bar{\partial}\left(\frac{1}{x}\right) \wedge \operatorname{tr}\left[\left[\eta_{-}, \bar{\alpha}_{+}\right] \wedge \varphi_{b}\right]=0
\end{gathered}
$$

where we have used (14).

Thus, the corresponding six-dimensional action of the localized modes on the matter curve $\Sigma: x=0$ is given by

$$
\begin{aligned}
S_{\Sigma}= & \pi \int d^{4} x \int_{\Sigma} i d y \wedge \bar{y} \sqrt{h_{y \bar{y}}}\left[-\left(\partial_{\mu} \zeta_{+}\right)^{\dagger}\left(\partial^{\mu} \zeta_{+}\right)-\left(\partial_{\mu} \zeta_{-}\right)^{\dagger}\left(\partial^{\mu} \zeta_{-}\right)\right. \\
& \left.-2 h^{y \bar{y}}\left(\left(D_{\bar{y}} \zeta_{+}\right)^{\dagger}\left(D_{\bar{y}} \zeta_{+}\right)+\left(D_{\bar{y}} \zeta_{-}\right)^{\dagger}\left(D_{\bar{y}} \zeta_{-}\right)\right)\right]
\end{aligned}
$$

where the covariant derivatives are given by

$$
D_{\bar{y}} \zeta_{ \pm}=\partial_{\bar{y}} \zeta_{ \pm} \pm 2 \bar{\alpha}_{3 y} \zeta_{ \pm}
$$

Let us proceed to the $\mathbf{Z}_{2}$ monodromy background, which was called a T-brane in Ref. 18. We will consider it in an $S U(3)$ gauge theory, and the background in the 
flat metric (12) is then given by

$$
\phi=\left(\begin{array}{lll}
0 & 1 & \\
x & 0 & \\
& & 0
\end{array}\right), \quad H=\left(\begin{array}{lll}
\sqrt{r} e^{\sigma(r)} & \\
& \frac{1}{\sqrt{r}} e^{-\sigma(r)} & \\
& & 1
\end{array}\right)
$$

with $r=|x|$, where the function $\sigma$ depends only on $r$ and satisfies the Painlevé III equation

$$
\left(\frac{d^{2}}{d s^{2}}+\frac{1}{s} \frac{d}{d s}\right) \sigma=\frac{1}{2} \sinh (2 \sigma)
$$

with $s=8 r^{3 / 2} /\left(3 \sqrt{h_{y \underline{y}}}\right)$. The solution has been given in Ref. 21 , and, near the origin $x=0$, it behaves as

$$
\sqrt{r} e^{\sigma} \simeq K\left(h_{y \bar{y}}\right)^{\frac{1}{6}}(1+\cdots),
$$

with a constant $K$. It therefore gives

$$
H(r) \simeq\left(\begin{array}{lll}
K\left(h_{y \bar{y}}\right)^{\frac{1}{6}} & & \\
& \frac{1}{K\left(h_{y \bar{y}}\right)^{\frac{1}{6}}} & \\
& & 1
\end{array}\right)
$$

Here note that $K$ is positive. The localized modes are located on the curve $\Sigma: x=0$, and they are given by

$$
h_{+}(y)=\left(\begin{array}{ccc}
0 & & \\
& 0 & \zeta_{+}(y) \\
& & 0
\end{array}\right), \quad h_{-}(y)=\left(\begin{array}{ccc}
0 & & \\
& 0 & \\
\zeta_{-}(y) & & 0
\end{array}\right) \text {, }
$$

along with

$$
\eta_{+}(y)=\left(\begin{array}{ccc}
0 & & \zeta_{+}(y) \\
& 0 & \\
& & 0
\end{array}\right), \quad \eta_{-}(y)=\left(\begin{array}{ccc}
0 & & \\
& 0 & \\
& -\zeta_{-}(y) & 0
\end{array}\right) .
$$

We will also concentrate on the localized mode $h_{+}(y)$. In this case, the condition (9) is equivalent to the D-term condition (8), and the solution to it for the mode $\varphi_{+}(y)$ has been discussed in Ref. 18 near the matter curve $\Sigma: x=0$ and far away from it. In fact, near the curve $\Sigma$, it is given by

$$
v_{+} \simeq \eta_{+}(y) \frac{1}{x}\left(B e^{-\frac{1}{K}\left(\frac{1}{h_{y \bar{y}}}\right)^{\frac{2}{3}}|x|^{2}}+C e^{+\frac{1}{K}\left(\frac{1}{h_{y \bar{y}}}\right)^{\frac{2}{3}}|x|^{2}}-1\right),
$$

where the constants $B$ and $C$ should be determined by the boundary condition at points far away from the matter curve $\Sigma: x=0$ and satisfy that $B+C=1$. It has 
been argued that $B$ is almost equal to unity. Therefore obtaining

$$
\left.\bar{\partial} v_{+}\right|_{x=0} \simeq-\frac{1}{K}\left(\frac{1}{h_{y \bar{y}}}\right)^{\frac{2}{3}} \eta_{+}(y) d \bar{x} .
$$

One thus obtains the normalization $\mathcal{N}_{+}$as

$$
\mathcal{N}_{+} \simeq \pi \int_{\Sigma}\left|\zeta_{+}(y)\right|^{2} \sqrt{h_{y \bar{y}}} i d y \wedge d \bar{y}
$$

For the bulk modes,

$$
\bar{\alpha}=\frac{1}{\sqrt{6}}\left(\begin{array}{ccc}
\bar{\alpha}_{8} & & \\
& \bar{\alpha}_{8} & \\
& & -2 \bar{\alpha}_{8}
\end{array}\right)+\left(\begin{array}{ccc} 
& 0 & \\
\bar{\alpha}_{2} & & \\
& & 0
\end{array}\right),
$$

the superpotential yields the Yukawa coupling with the localized modes $\zeta_{ \pm}$,

$$
\begin{aligned}
& -i \pi \int \delta^{2}(x) d \bar{x} \wedge\left[\operatorname{tr}\left[h_{+} \wedge\left[\bar{\alpha}_{b}, \eta_{-}\right]\right]+\operatorname{tr}\left[h_{-} \wedge\left[\bar{\alpha}_{b}, \eta_{+}\right]\right]\right] \\
& \quad=-\sqrt{6} i \pi \int \delta^{2}(x) d \bar{x} \wedge \zeta_{+} \bar{\alpha}_{8} \zeta_{-} d x \wedge d y=\sqrt{6} \pi \int \zeta_{+} \bar{\alpha}_{8 \bar{y}} \zeta_{-} i d y \wedge d \bar{y}
\end{aligned}
$$

and

$$
\begin{gathered}
\int \operatorname{tr}\left[\left(\bar{\alpha}_{+} \wedge \bar{\alpha}_{-}+\bar{\alpha}_{-} \wedge \bar{\alpha}_{+}\right) \wedge \varphi_{b}\right]=\int \operatorname{tr}\left[\left(\bar{\alpha}_{+} \wedge \bar{\partial} v_{-}+\bar{\partial} v_{-} \wedge \bar{\alpha}_{+}\right) \wedge \varphi_{b}\right] \\
=-\int \bar{\partial}\left(\frac{1}{x}\right) \wedge \operatorname{tr}\left[\left[\eta_{-}, \bar{\alpha}_{+}\right] \wedge \varphi_{b}\right]=0
\end{gathered}
$$

where we have used (16).

The action of the localized modes $\zeta_{ \pm}$is also given by (15) with the covariant derivatives

$$
D_{\bar{y}} \zeta_{ \pm}=\partial_{\bar{y}} \zeta_{ \pm} \pm \sqrt{3} \bar{\alpha}_{8 y} \zeta_{ \pm}
$$

\section{Acknowledgments}

I am grateful to Yoichi Tsuchiya and Taizan Watari for the collaboration of this work. This work was supported in part by a Grant-in-Aid \#23540286 from the MEXT of Japan.

\section{References}

1. A. Anisimov, M. Dine, M. Graesser and S. D. Thomas, "Brane world SUSY breaking from string/M theory," JHEP 0203, 036 (2002) hep-th/0201256. O. DeWolfe and S. B. Giddings, "Scales and hierarchies in warped compactifications and brane worlds," Phys. Rev. D 67, 066008 (2003) hep-th/0208123. S. Kachru, R. Kallosh, A. D. Linde, J. M. Maldacena, L. P. McAllister and S. P. Trivedi, "Towards inflation in string theory," JCAP 0310, 013 (2003) hep-th/0308055. M. Grana, T. W. Grimm, H. Jockers and J. Louis, "Soft supersymmetry breaking in Calabi-Yau orientifolds 
with D-branes and fluxes," Nucl. Phys. B 690, 21 (2004) hep-th/0312232. D. Lust, P. Mayr, R. Richter and S. Stieberger, "Scattering of gauge, matter, and moduli fields from intersecting branes," Nucl. Phys. B696, 205-250 (2004) hep-th/0404134. Ref..? S. B. Giddings and A. Maharana, "Dynamics of warped compactifications and the shape of the warped landscape," Phys. Rev. D 73, 126003 (2006) hep-th/0507158. D. Baumann, A. Dymarsky, I. R. Klebanov, J. M. Maldacena, L. P. McAllister and A. Murugan, "On D3-brane Potentials in Compactifications with Fluxes and Wrapped D-branes," JHEP 0611, 031 (2006) hep-th/0607050. S. Kachru, L. McAllister and R. Sundrum, "Sequestering in String Theory," JHEP 0710, 013 (2007) hep-th/0703105, and many other works.

2. H. Hayashi, T. Kawano, Y. Tsuchiya and T. Watari, "Flavor Structure in F-theory Compactifications," JHEP 1008, 036 (2010) arXiv:0910.2762.

3. J. J. Heckman and C. Vafa, "Flavor Hierarchy From F-theory," Nucl. Phys. B 837, 137 (2010) arXiv:0811.2417.

4. S. Cecotti, M. C. N. Cheng, J. J. Heckman and C. Vafa, "Yukawa Couplings in Ftheory and Non-Commutative Geometry," arXiv:0910.0477.

5. J. Marsano, N. Saulina and S. Schafer-Nameki, "F-theory Compactifications for Supersymmetric GUTs," JHEP 0908, 030 (2009) arXiv:0904.3932.

6. C. Cordova, "Decoupling Gravity in F-Theory," Adv. Theor. Math. Phys. 15, 689 (2011) arXiv:0910.2955.

7. R. Tatar, Y. Tsuchiya and T. Watari, "Right-handed Neutrinos in F-theory Compactifications," Nucl. Phys. B 823, 1 (2009) arXiv:0905.2289.

8. H. Hayashi, T. Kawano, Y. Tsuchiya and T. Watari, "More on Dimension-4 Proton Decay Problem in F-theory - Spectral Surface, Discriminant Locus and Monodromy," Nucl. Phys. B840, 304-348 (2010) arXiv:1004.3870.

9. T. W. Grimm and T. Weigand, "On Abelian Gauge Symmetries and Proton Decay in Global F-theory GUTs," Phys. Rev. D82, 086009 (2010) arXiv:1006.0226.

10. J. Marsano, N. Saulina and S. Schafer-Nameki, "A Note on G-Fluxes for F-theory Model Building," JHEP 1011, 088 (2010) arXiv:1006.0483.

11. H. Hayashi, T. Kawano and T. Watari, "Constraints on GUT 7-brane Topology in F-theory," Phys. Lett. B 708, 191 (2012) arXiv:1112.2032.

12. S. Katz and C. Vafa, "Matter From Geometry," Nucl. Phys. B 497, 146 (1997) hepth/9606086.

13. G. Curio, "Chiral matter and transitions in heterotic string models," Phys. Lett. B 435, 39 (1998) hep-th/9803224.

14. D. E. Diaconescu and G. Ionesei, "Spectral covers, charged matter and bundle cohomology," JHEP 9812, 001 (1998) hep-th/9811129.

15. R. Donagi, Y. H. He, B. A. Ovrut and R. Reinbacher, "The particle spectrum of heterotic compactifications," JHEP 0412, 054 (2004) hep-th/0405014.

16. R. Blumenhagen, S. Moster, R. Reinbacher and T. Weigand, "Massless spectra of three generation U(N) heterotic string vacua," JHEP 0705, 041 (2007) hep-th/0612039.

17. H. Hayashi, R. Tatar, Y. Toda, T. Watari and M. Yamazaki, "New Aspects of Heterotic-F Theory Duality," Nucl. Phys. B 806, 224 (2009) arXiv:0805.1057.

18. S. Cecotti, C. Cordova, J. J. Heckman and C. Vafa, "T-Branes and Monodromy," JHEP 1107, 030 (2011) arXiv:1010.5780.

19. Martijn Wijnholt, private communication.

20. T. Kawano, Y. Tsuchiya and T. Watari, "A Note on Kahler Potential of Charged Matter in F-theory," Phys. Lett. B 709, 254 (2012) arXiv:1112.2987.

21. B. M. McCoy, C. A. Tracy and T. T. Wu, "Painleve Functions of the Third Kind," J. Math. Phys. 18, 1058 (1977).

A. B. Zamolodchikov, "Painleve III and 2-d Polymers," Nucl. Phys. B 432, 427 (1994) hep-th/9409108. 